\title{
Evaluation of endothelial function using finger plethysmography
}

Faizi AK, MD, Kornmo DW, MD, Agewall S, MD, Ph.D.

Department of Medicine, Aker University Hospital and Oslo University, 0514 Oslo, Norway

Correspondence: Stefan Agewall, Department of Medicine, Aker University Hospital and Oslo University, 0514 Oslo, Norway. E-mail: stefan.agewall@ medisin.uio.no.

Tel +4722894655. Fax +4722894259 


\section{ABSTRACT}

\section{Background:}

Previous studies have used upper-arm with 5 minutes cuff occlusion for measuring reactive hyperemic index (RHI). The aims of the present study are, to establish the optimum duration of blood flow occlusion to obtain maximal response, and to compare the response after lower arm and upper arm occlusion.

Objective: To establish the optimum duration of blood flow occlusion, which results in maximal response in form of reactive hyperemia $(\mathrm{RH})$ and to compare the responses after forearm and upper arm occlusion of young healthy adults.

Method: Pulse wave amplitude (PWA) using a novel finger plethysmograph (EndoPat, Itamar). For measuring RH induced by forearm cuff occlusion, each subject was examined at different days in a random order of four cuff occlusion times (1.5, 3, 5, and 8 minutes). RH induced by five minutes upper arm cuff occlusion was also measured in 20 subjects. Subjects included 13 male and 17 female subjects with mean age 33 years (range from 22 to 50) with no known risk factors for cardiovascular disease, being on no regular pharmacological treatment, non diabetic, fasting for 12 hours and not being pregnant in case of females. Main outcome measured RHI (reactive hyperemic index; calculated as the ratio of the digital pulse volume during reactive hyperemia divided by that at baseline). Mean value of RHI of the different occlusion times in the 
forearm were compared. The response after 5 minutes forearm occlusion time was compared with same occlusion time response in the upper arm.

Results: Average RHI was lower with 1.5 and 3 minutes forearm occlusion as compared to 8 minutes forearm occlusion (1.52 vs $1.94: p=0.002,1.63$ vs $1.94: p=0.024)$. No significant difference between values of 5 minutes and 8 minutes forearm occlusion $(1.88$ vs $1.94: p=$ 0.357). No significant difference found between 5 minutes forearm and 5 minutes over arm (1.88 vs $2.07: p=0.097)$

Conclusion: Measures of endothelial function using finger plethysmography vary with duration of blood flow occlusion. Maximum response was reached after 5 minutes of blood flow occlusion and therefore this occlusion time is recommended. The response after forearm and upper arm occlusion did not differ significantly. Both positions are possible to use, but the cuff position needs to be standardized in an individual study. Forearm occlusion might be preferred since this occlusion caused less discomfort.

\section{INTRODUCTION}

The endothelium synthesizes and releases several vasoactive factors, including nitric oxide, which plays an important role in maintaining normal endothelial function. ${ }^{1}$ A variety of disorders can decrease nitric oxide function resulting in endothelial dysfunction which is an important factor in atherogenesis.$^{2}$ Impaired endothelium dependent relaxation has been observed in subjects with atherosclerosis. ${ }^{3,4}$ Endothelial dysfunction predict cardiovascular disease ${ }^{5,6}$ If 
endothelial dysfunction can be identified prior to the development of atherosclerosis, interventions to prevent atherosclerosis may be used before the development of clinical disease.

The gold standard technique for measuring endothelial function in clinical studies is Flow Mediated Dilatation (FMD) of the brachial artery. ${ }^{7}$ Recently it has been demonstrated that abnormalities in pulse wave amplitude (PWA) using a novel finger plethysmograph (peripheral arterial tonometry $[\mathrm{PAT}])$ is significantly associated with FMD. ${ }^{8}$ By this method beat to beat plethysmographic measurement of the finger arterial pulse wave is performed. Main outcome measures reactive hyperemic index (RHI), calculated as the ratio of the digital pulse volume during reactive hyperemia divided by that at baseline. ${ }^{9} \mathrm{RHI}$ is shown to be attenuated in patients with coronary microvascular endothelial dysfunction, suggesting a role for PAT as noninvasive test to identify patients with coronary disorder. Whereas the FMD technique is rather standardized, ${ }^{7}$ the finger plethysmograph technique needs to be further investigated.

Previous studies have used upper-arm with 5 minutes cuff occlusion for measuring RHI. The aims of the present study are, to establish the optimum duration of blood flow occlusion to obtain maximal response, and to compare the response after lower arm and upper arm occlusion.

\section{METHODS:}

Thirty adult volunteers (17 female, 13 male). Fifteen subjects between the age of 20-30 and fifteen subjects between the ages of 40-50. The younger subjects (20-30) were recruited from our university and middle-aged subjects (40-50) from the university hospital as well as office workers 
outside the hospital environment. All were normotensive, non-smokers, no history of diabetes or cardiovascular disease, fasting for 12 hours and not being pregnant in case of females.

All subjects were measured for reactive hyperemia, induced by forearm cuff occlusion. Each subject was examined at different days in a random order of four cuff occlusion times $(1.5,3,5$, and 8 minutes). Ten subjects from each age group also underwent RH-PAT measurement after 5 minutes upper-arm cuff occlusion.

Finger pulse wave amplitude (PWA) was recorded with PAT (EndoPAT, Itamar Medical Ltd, Caesarea, Isreal). PAT is a noninvasive technology that captures a beat-to-beat plethysmographic recording of the finger arterial PWA with pneumatic probes. ${ }^{8}$ The PAT finger probe consists of a thimble-shaped sensor cap that imparts a uniform pressure field and exhibits a clamp-like effect on the entire surface of the distal phalanx and measures pulsatile volume changes. PAT applies a significant counterpressure $(70 \mathrm{~mm} \mathrm{Hg})$ on the digit and avoids distal venous distention, thereby inhibiting venous pooling and blood stasis, which could otherwise induce a venoarteriolar reflex vasoconstrictor response. The pressure field applied to the finger may also protect against local venous distension related to elevated venous pressure in the upper arm during the cuff inflation portion of reactive hyperemia testing. ${ }^{8}$ PAT is thus configured to unload arterial wall tension and increase the range of arterial wall motion without inducing potentially confounding vasomotor changes. The finger probe is connected by flexible tubing to isolated volume reservoirs that buffer pressure changes within the probes. The pressure change signals are then filtered, amplified, displayed, and stored for further analysis. 
Peripheral arterial tonometry probes were placed on one finger of each hand for continuous recording of the PAT signal. After an 8-min equilibration period which is used as baseline, the blood pressure cuff was inflated to suprasystolic pressures for 1.5, 3, 5, $8 \mathrm{~min}$. Then the cuff was deflated, while PAT recording continued for 10 minutes. Main outcome measured reactive hyperemic index (RHI), calculated as the ratio of the digital pulse volume during reactive hyperemia over a 1-min time interval starting 1 min after cuff deflation divided by that at baseline (3.5-min time period before cuff inflation). ${ }^{9}$ This study was approved by the Norwegian Regional Ethic Committee (REK).

\section{RESULTS:}

The characteristics of the study population are listed in Table 1. Average RHI was lower with 1.5 and 3 minutes forearm occlusion as compared to 8 minutes forearm occlusion (1.52 vs 1.94 : $p=0.002,1.63$ vs $1.94: p=0.024)($ Figure 1$)$. There was no significant difference found between values of 5 minutes and 8 minutes forearm occlusion (1.88 vs $1.94: p=0.357)$. Figure 3 and 4 show the corresponding values in the young and middle-aged groups. In Figure 4 average RHI as percentage of the RHI after 8 minutes occlusion is given. We found no significant difference between 5 minutes forearm and 5 minutes over arm (1.88 vs $2.07: p=0.097)$ (Figure 5). Comparison between upper-arm and forearm occlusion are in middle-aged (Figure 6) and young group (Figure 7). In the middle aged group there was no difference found. There was significant difference between forearm and upper-arm values in younger group where upper-arm occlusion tended to cause higher RHI than with forearm $(p=0.012)$. All subjects reported that forearm occlusion was less unpleasant compared to upper-arm occlusion. 


\section{DISCUSSION:}

This study aimed to establish the optimum duration of blood flow occlusion to obtain maximal response, and to compare the response after lower arm and upper arm occlusion using the novel finger plethysmograph (peripheral arterial tonometry [PAT]) technique. ${ }^{8}$ Maximum response was reached after 5 minutes of blood flow occlusion and therefore this occlusion time should be recommended. The response after forearm and upper arm occlusion did not differ significantly. Both positions are possible to use, but the cuff position needs to be standardized in an individual study. Forearm occlusion might be preferred since this occlusion caused less discomfort.

Hyperemia after active ischemia has been tried and studied as means of understanding the endothelial function. This is done with point of view that reactive hyperemia is dependent on production of nitric oxide (NO) by the endothelium. ${ }^{1}$ Previously these studies have been performed with invasive means, either by measuring the intra-venous concentration of NO before and after active ischemia in subjects or by direct instrumentation of the small arteries. During the last decade FMD has become a standardized non-invasive measure of endothelial function. ${ }^{9}$ However, the FMD technique requires a very experienced ultrasonographer. In the guideline it is stated that at least 100 supervised scans and measurements should be performed before independent scanning and reading is attempted and at least 100 scans per year should be performed to maintain competency. Therefore, there is a need for new and simple to perform methods to measure endothelial function in a non-invasive procedure.

Assessment of peripheral vascular endothelial function with finger arterial pulse wave amplitude (PWA) demonstrates similar pattern to that of brachial artery ultrasound assessment of FMD. 
This supports the concept that analysis of PWA with PAT during reactive hyperemia maybe used to study peripheral vascular endothelial dysfunction. ${ }^{8}$ This technique has been used in different studies to show coronary microvascular endothelial dysfunction by comparing healthy individuals with patients having coronary endothelial dysfunction. Five minutes upper-arm cuff occlusion has been used as occlusion time in these studies. ${ }^{8,9}$ Our study is a part of further assessment of this method, and its eventual possible use as a noninvasive test to identify patients with endothelial disorder.

In this study we tried to find if there are any significant differences in RHI with different time intervals of forearm cuff occlusion. At the same time we compared the five minutes over-arm cuff occlusion which has been shown to give maximum arterial response by ultrasound assessment, ${ }^{11}$ with five minutes forearm occlusion in our study. We found that forearm can be used as substitute for over-arm cuff occlusion and at the same time causes less discomfort compared to upper-arm. Average RHI was lower with 1.5 and 3 minutes forearm occlusion as compared to 8 minutes forearm occlusion. No significant difference was found between values of 5 minutes and 8 minutes forearm occlusion and neither between 5 minutes forearm and 5 minutes over arm. Most previous studies using this technique have used upper-arm occlusion. ${ }^{8,9,13}$

The observation that upper arm occlusion during FMD examination has been followed by a more pronounced dilatation of the brachial artery compared with after forearm occlusion has been previously reported. ${ }^{7,12}$ The local ischaemia of the brachial artery with a proximal occlusion may explain why the brachial artery dilated more after upper arm occlusion compared with after forearm occlusion. As far as we know there are no such studies comparing different occlusion 
positions using the finger plethysmograph (peripheral arterial tonometry [PAT]) technique. We did not observe any significant difference between 5 minutes forearm and 5 minutes upper arm occlusion, but there was a tendency to a more pronounced response after upper arm occlusion, in line with the previous reports from FMD examinations. ${ }^{7,12}$ Thus, it appears to be possible to use both upper-arm and fore- arm occlusions but it must clearly be stated which of the methods that was used. We observed that upper-arm occlusion according to the study subjects was more unpleasant compared to fore-arm occlusion and therefore forearm occlusion might be preferred in clinical studies.

\section{Conclusion:}

Maximum response was reached after 5 minutes of blood flow occlusion and therefore this occlusion time should be recommended when using the novel finger plethysmograph (peripheral arterial tonometry [PAT]) technique. The response after forearm and upper arm occlusion did not differ significantly. Both positions are possible to use, but the cuff position needs to be standardized in an individual study. Forearm occlusion might be preferred since this occlusion caused less discomfort. 


\section{REFERENCES}

1. Furchgott R. F. Zawadzki J. V. The obligatory role of endothelial cells in the relaxation of arterial smooth muscle by acetylcholine. Nature. 1980; 299:373-6.

2. Ross R. The pathogenesis of atherosclerosis: a perspective for the 1990s. Nature. 1993;362: 801 - 9

3. Liebermane H., Gerhard M. D., Uehata A., et al. Flow-induced vasodilatation of the human brachial artery is impaired in patients <40 years of age with coronary artery disease. Am J Card, 1996; 78: 1210-14.

4. Ludmer P. L., Selwyn A. P., Shook T. L., et al. Paradoxical vasoconstriction induced by acetylcholine in atherosclerotic coronary arteries. N Engl J Med, 1986;315: 1046-51

5. Gokce N, Keaney JF, Hunter LM, et al. Risk stratification for postoperative cardiovascular events via noninvasive assessment of endothelial function: a prospective study. Circulation. 2002; 105: 1567-72

6. Gregorio Brevetti, Antonio Silvestro, Vittorio Schiano and Massimo Chiariello. Circulation 2003; 108: 2093-98; originally published online Oct 6, 2003;

7. Agewall.S, R. N. Doughty, W. Bagg, et al. Comparison of ultrasound assessment of Flow-mediated dilatation in the radial and brachial artery with upper and forearm cuff positions. Clinical Physiology 2001;21: 9-14

8. Jeffrey T. Kuvin, Ayan R. Patel, MD, et al. Assessment of peripheral vascular endothelial function with finger arterial pulse wave amplitude. American Heart Journal. 2003; 146:161-72

9. Piero O. Bonetti, MD, Geralyn M,et al. Noninvasive Identification of Patients with Early Coronary Atherosclerosis by Assessment of Digital Reactive Hyperemia . J. Am. Coll. Cardiol. 2004; 44: 2137-41 
10. Corretti MC, Anderson Todd J., MD, Benjamin Emelia J. Guidelines for the Ultrasound Assessment of Endothelial-Dependent Flow-Mediated Vasodilation of the Brachial Artery. J Am Coll Cardiol 2002;39: 257-65

11. P. Leeson, S. Thorne, A. Donald et al. Non-invasive measurement of endothelial function: effect on brachial artery dilatation of graded endothelial dependant and independent stimuli. Heart 1997;78:22-7

12. Corretti MC, Plotnick GD, Vogel RA. Technical aspects of evaluating brachial artery vasodilatation using highfrequency ultrasound. Am J Physiol, 1995; 268: 1397-404

13. Lowenstein Lior, Damti Amit, Pillar Giora, et al. European Journal of Obstetrics \& Gynecology and Reproductive Biology, 2007; 134:208-12 


\section{Figure Legends}

Figure 1: Forearm cuff occlusion with $1.5,3,5$ and 8 minutes. (*) Shows duration of cuff occlusion where RHI is significantly different $(\mathrm{P}=0.05)$ from $\mathrm{RHI}$ after eight minutes.

Figure $2 \&$ 3: Forearm cuff occlusion with 1.5, 3, 5 and 8 minutes in young and middle-aged groups.

Figure 4: RHI as percent of 8 minutes cuff occlusion. (*)Shows duration of cuff occlusion where the RHI is significantly different $(\mathrm{P}=0.05)$ from $\mathrm{RHI}$ after eight minute.

Figure 5: Five minutes forearm and upper arm cuff occlusion. There was no significant difference found.

Figure 6 and 7: Five minutes forearm and upper-arm cuff occlusion in middle-aged and young groups. 
Table 1: Characteristics of study groups (mean (1 SD))

\begin{tabular}{lcclll} 
Age(years) & Number & Gender & HR & Systolic BP & Diastolic BP \\
\hline Young 24 (1.54) & 15 & $\delta(8)$, o $(7)$ & $60(9.9)$ & $119(12.4)$ & $70(6.8)$ \\
Middle-aged 43(2.91) & 15 & $\delta(5)$, o $(10)$ & $72(8.6)$ & $128(10.9)$ & $76(15.5)$
\end{tabular}




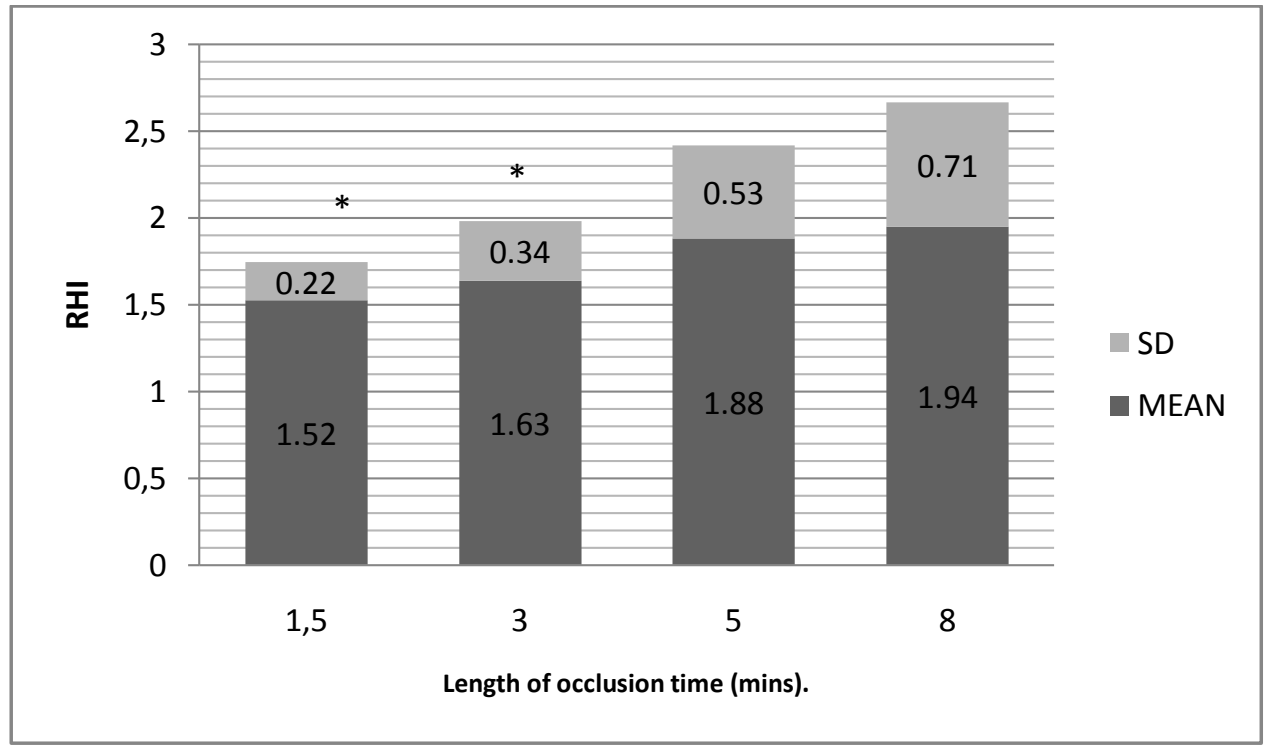

Figure 1:

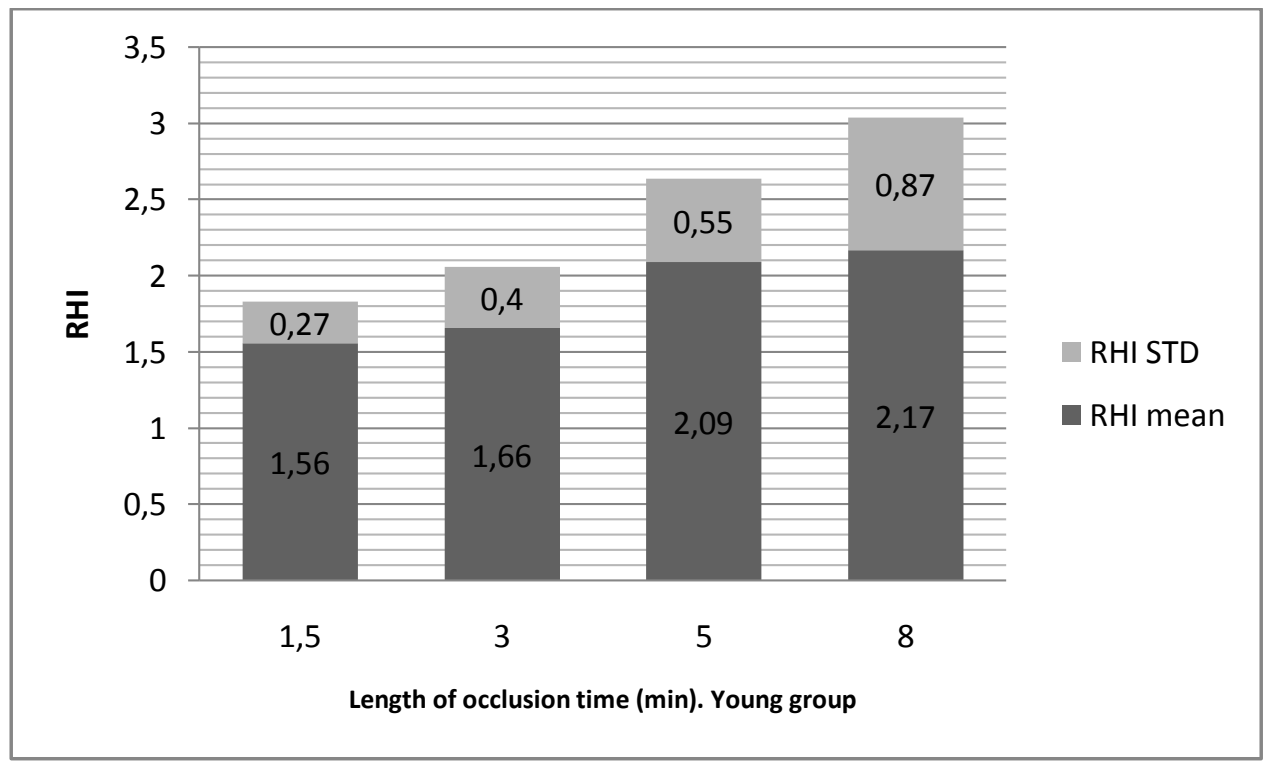

Figure 2: 


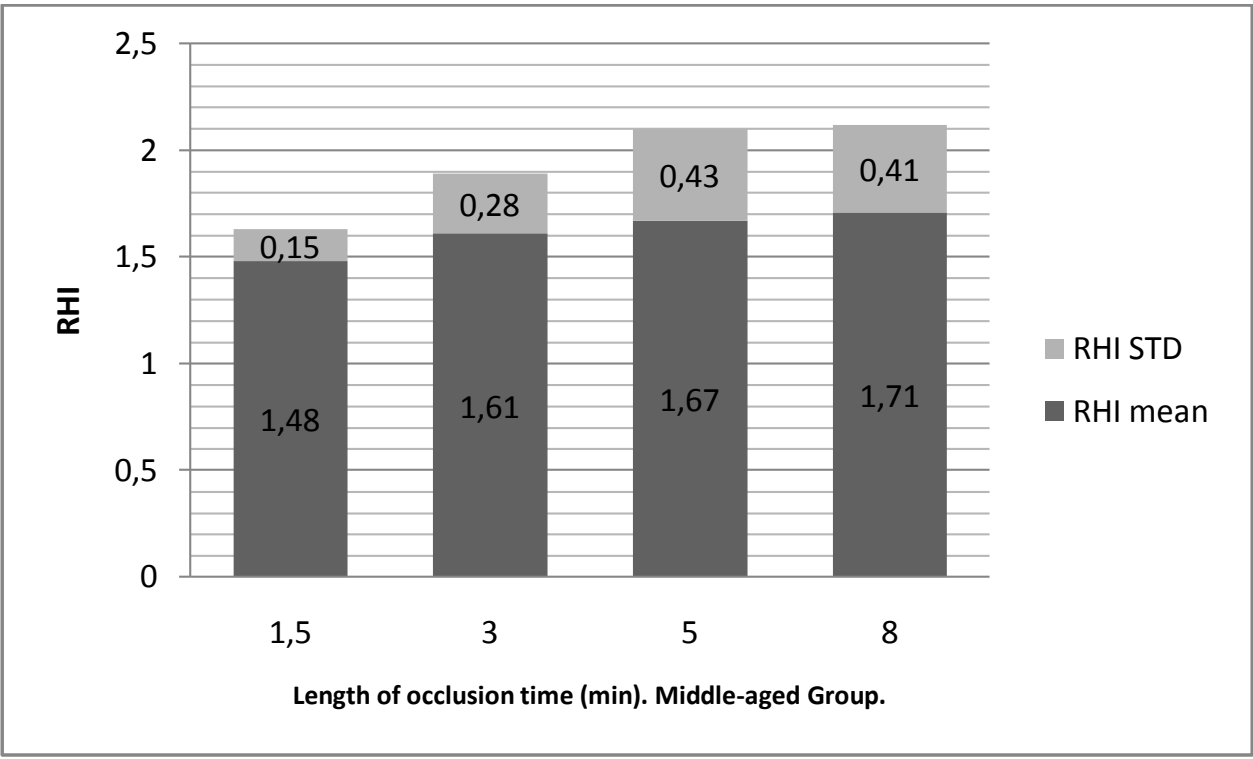

Figure 3:

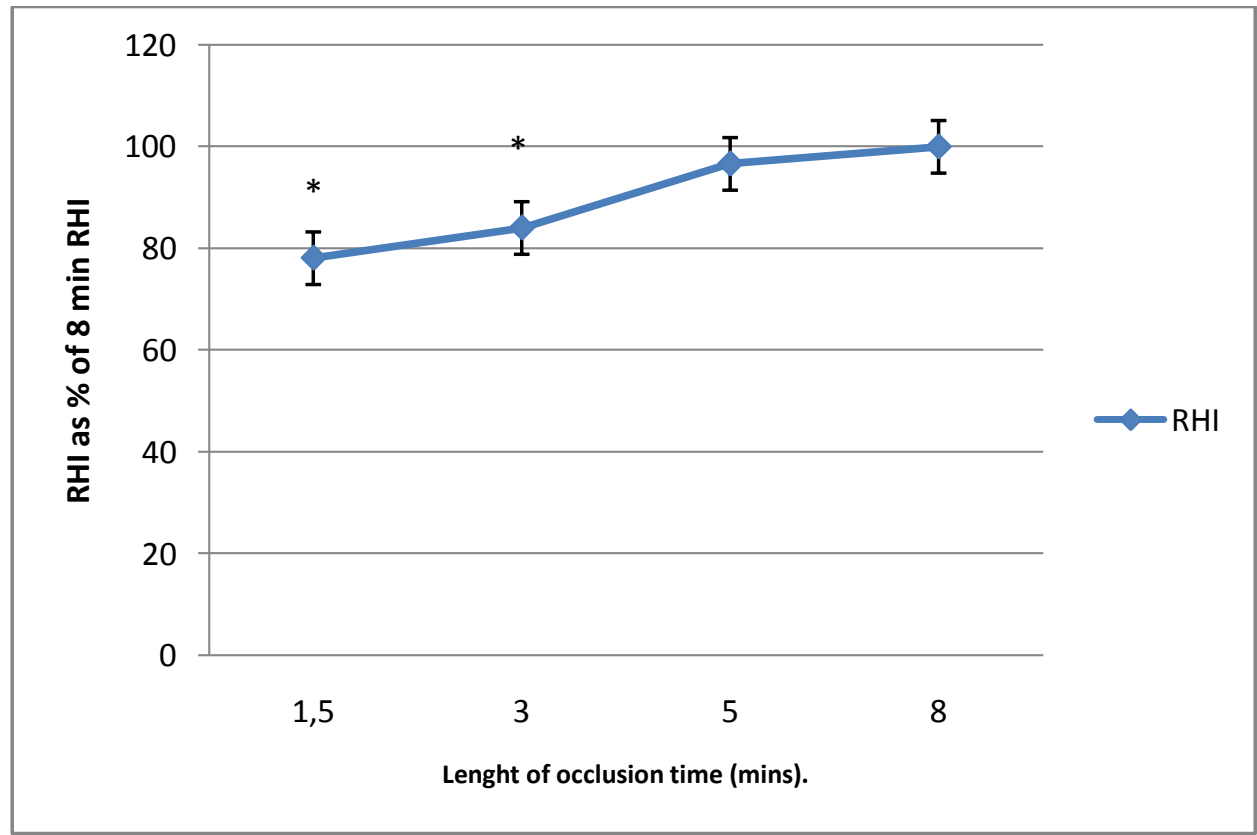

Figure 4: 


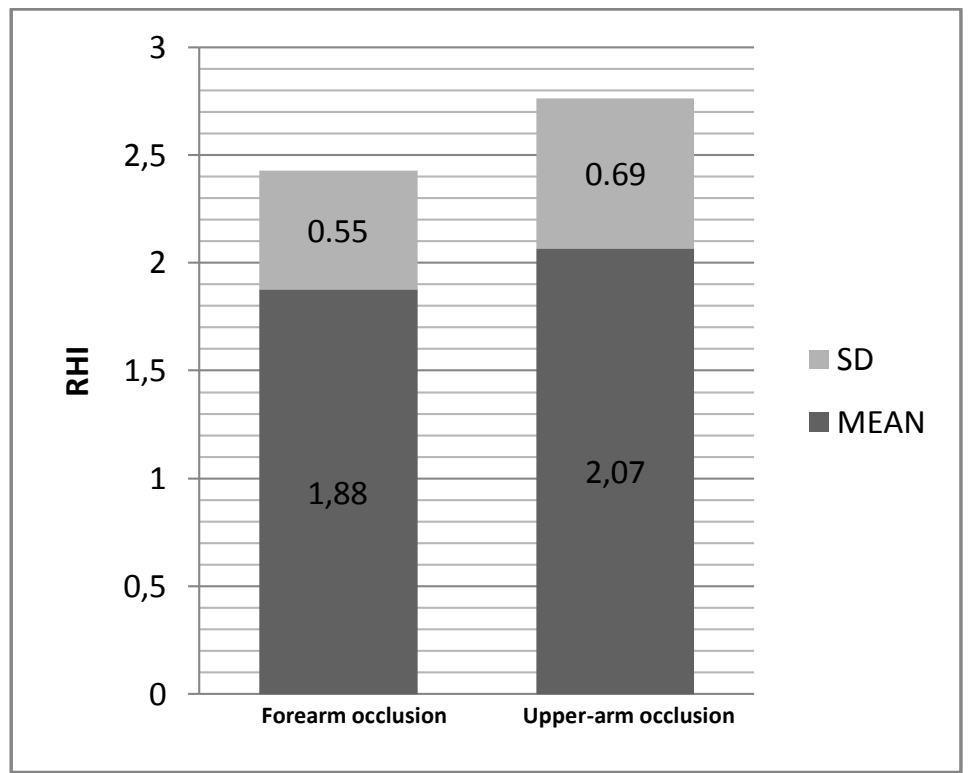

Figure 5:

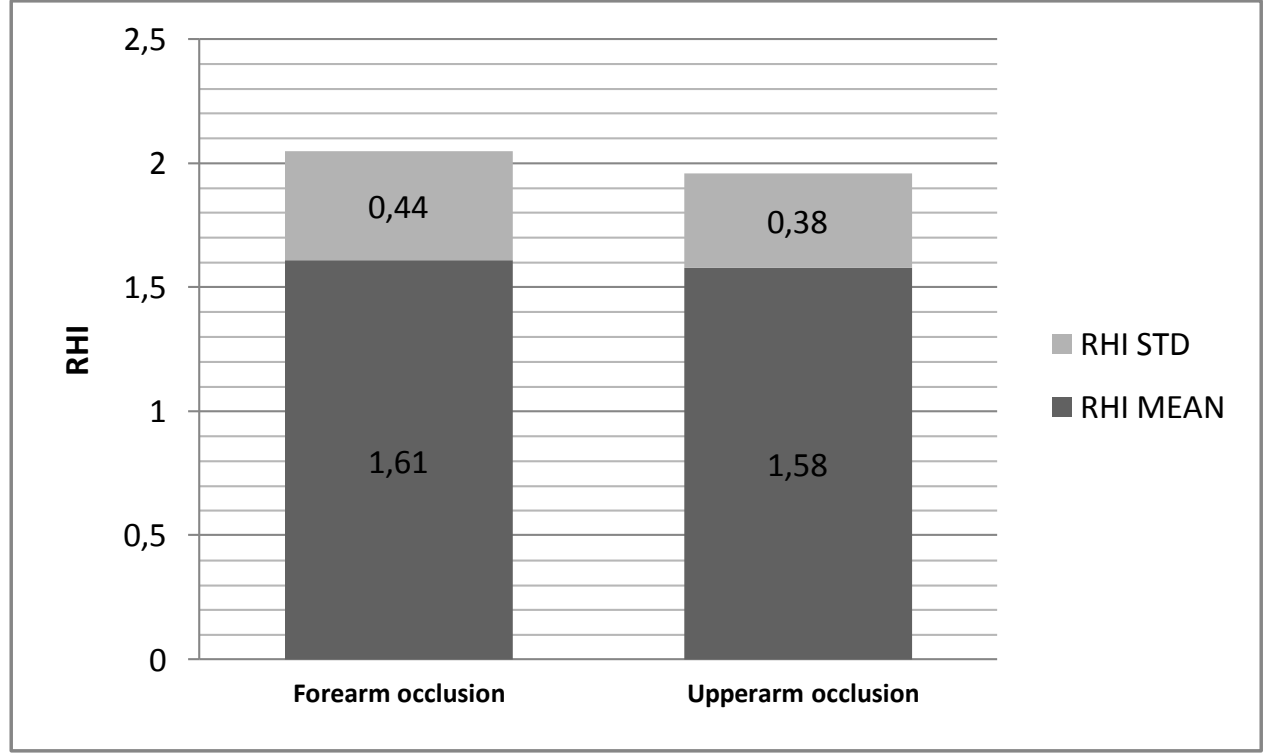

Figure 6: 


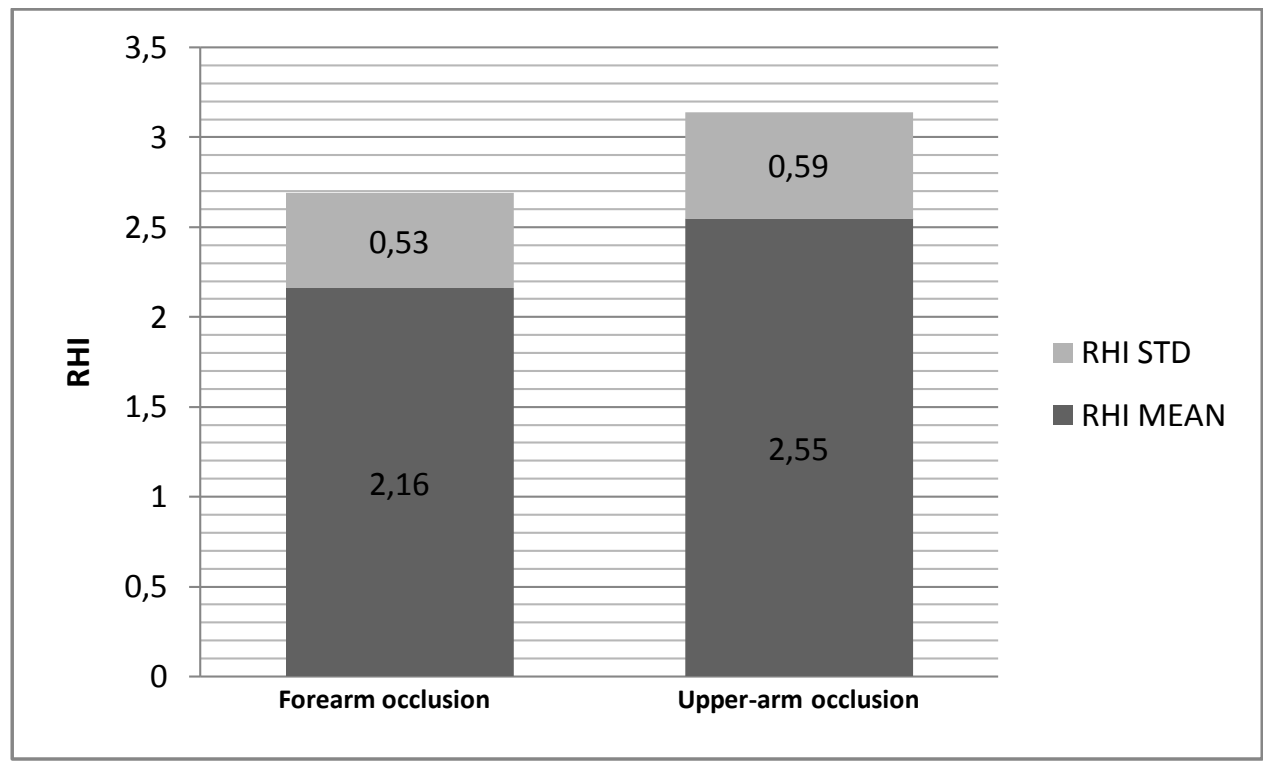

Figure 7: 